\title{
Fractional-order feedback control of a poorly damped system
}

\author{
Amélie Chevalier, Cosmin Copot, Dana Copot, Clara M. Ionescu, Robin De Keyser \\ Ghent University, Department of Electrical energy, Systems and Automation (EeSA), \\ Technologiepark 913, 9052, Gent-Zwijnaarde, Belgium. \\ Email: \{amelie.chevalier, cosmin.copot, dana.copot, claramihaela.ionescu, robain.dekeyser\}@ugent.be
}

\begin{abstract}
This study presents the design of a fractionalorder proportional-integral (FOPI) controller for a mass-springdamper system which is poorly damped. A model based design technique is used to design a FOPI controller for this system. A good performance of the closed loop control of a high order oscillatory system, such as the mass-spring-damper system, is with traditional proportional-integral (PI) controllers difficult to achieve. Therefore, a comparison between a traditional PI controller and a FOPI controller is performed by simulation. The simulation results show that the FOPI controller outperforms the classical PI controller resulting in an increased damping of the oscillations while maintaining a reasonable control effort.
\end{abstract}

Keywords-Fractional-order control, PI control, poorly damped system, mass spring damper

\section{INTRODUCTION}

The mass-spring-damper system is a classical example of an electromechanical system. Its applications are manifold and include drives [1], active suspensions [2] but also biomedical applications such as modeling the human body [3] and applications in sound [4].

The classical mass-spring-damper system is a challenging system as each mass-spring construction introduces a peak in the frequency response of the system, resulting in resonance frequencies and high oscillations if damping is poor (like the case in this paper). Traditionally, these kind of systems are difficult to control by an integer-order proportional-integralderivative (PID) controller as this controller has only one pair of zeros to compensate the system. Therefore, a controller of higher order would be more suitable to control poorly damped systems such as the mass-spring-damper. Advanced controllers such as fractional-order controllers may be better but also more complex as they can be approximated by high order integerorder transfer functions.

In the last decades there has been an increasing interest in fractional-order controllers. Applying fractional calculus techniques in the modeling and control of various dynamical systems is well recognized since Podlubny [5] proposed his extension of the integer-order PID controller. The superior performance of the fractional-order controllers over integerorder controllers has been shown on many occasions [6]. However, by adding two more design parameters to the classical integer-order PID controller, designing a fractional order PID controller can be challenging. As a result, the design and tuning of fractional-order controllers has been widely investigated such as auto-tuning of FOPID controllers [7], Ziegler-Nichols type of tuning rules for FOPID controllers [8] and tuning rules based on maximum sensitivity [9]. In its mathematical framework a fractional-order PID can be viewed by a finite number of pole-zero pairs, with a frequency interval [10]. This is one of the most commonly used methods of implementations in real life systems of such controllers. Intuitively, one expects that this may help to compensate for the exotic dynamics of a mass-spring-damper system.

This paper discusses the design of a fractional-order proportional-integral controller for a mass-spring-damper system. The performance of the fractional-order controller is compared to that of the integer-order controller for this poorly damped system.

The structure of the paper is as follows: the next section presents the mass-spring-damper system used in this research and derives the necessary transfer functions. Section three discusses the controller design. A first subsection presents the design of an integer-order PI controller using model-based computer aided design tools. The fractional-order PI controller is designed in the next subsection and is also based on model-based techniques. Section four discusses the performed simulations and the results. It presents the implementation method of fractional-order controllers and two simulations. In the first simulation the FOPI controller is compared to the classical PI controller. A second simulation investigates the robustness to gain variation of the system. A conclusion is formed in the final section.

\section{PLANT DESCRIPTION}

The mass spring damper system used in this paper (see Fig. 1) is an electromechanical system with two movable masses $m_{1}$ and $m_{2}$ (the third mass in the picture is fixed for this experiment), three springs with spring constants $k_{1}, k_{2}$ and $k_{3}$, a damper with damping constant $c_{1}$ and a motor which drives the system. The input of the system is the voltage to the motor $u(t)$ while the two outputs of the system are the mass displacements $y_{1}$ and $y_{2}$ expressed in $\mathrm{cm}$. As the dynamics of the electrical motor are much faster than those of the massspring-damper system, they can be neglected. Hence, the motor can be represented by a pure static gain $F(t)=K \cdot u(t)$, with $F(t)$ the force on the first mass. The parameters of the currently used setup are: $m_{1}=1.85 \mathrm{~kg}, m_{2}=1.35 \mathrm{~kg}$, $k_{1}=k_{2}=800 \mathrm{~N} / \mathrm{m}, k_{3}=450 \mathrm{~N} / \mathrm{m}, c_{1}=9 \mathrm{~N} /(\mathrm{m} / \mathrm{s})$ and $K=1 \mathrm{~N} / \mathrm{V}$. 

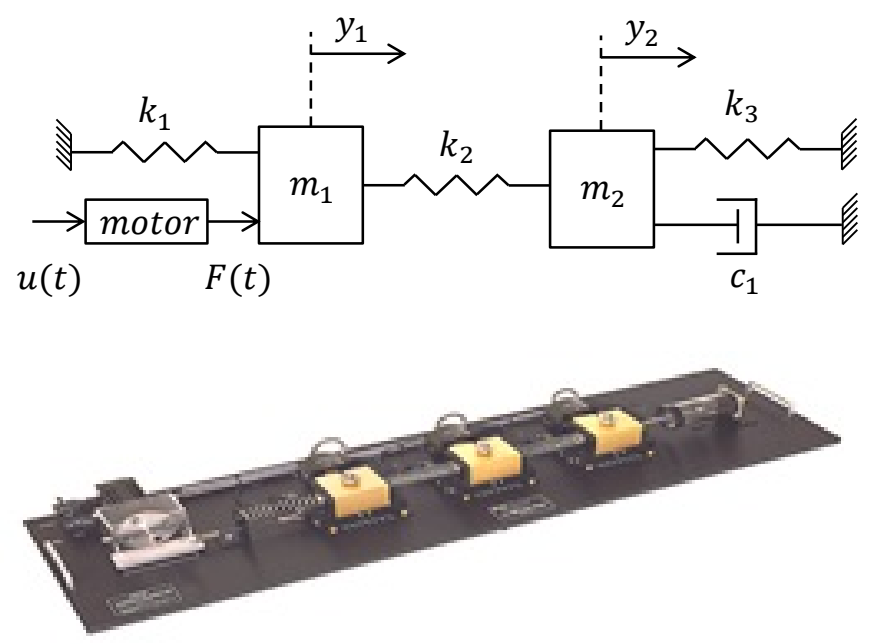

Fig. 1. Schematic representation and real life plant of the mass-spring damper system.

A complete model of the electromechanical plant describes the dynamics from $u(t)$ to $y_{1}(t)$ and from $u(t)$ to $y_{2}(t)$. The two differential equations describing the dynamics of the system are:

$$
\begin{gathered}
m_{1} \ddot{y}_{1}(t)+\left(k_{1}+k_{2}\right) y_{1}(t)=F(t)+k_{2} y_{2}(t) \\
m_{2} \ddot{y}_{2}(t)+c_{1} \dot{y}_{2}(t)+\left(k_{2}+k_{3}\right) y_{2}(t)=k_{2} y_{1}(t)
\end{gathered}
$$

After taking the Laplace transforms of equations (1) and (2), the resulting transfer functions are:

$$
\begin{gathered}
T F_{1}(s)=\frac{Y_{1}(s)}{U(s)}=\frac{K\left(m_{2} s^{2}+c_{1} s+\left(k_{2}+k_{3}\right)\right)}{d e n} \\
T F_{2}(s)=\frac{Y_{2}(s)}{U(s)}=\frac{K k_{2}}{d e n}
\end{gathered}
$$

with den $=m_{1} m_{2} s^{4}+m_{1} c_{1} s^{3}+$ $\left[m_{1}\left(k_{2}+k_{3}\right)+m_{2}\left(k_{1}+k_{2}\right)\right] s^{2}+c_{1}\left(k_{1}+k_{2}\right) s+k_{1} k_{2}+$ $k_{1} k_{3}+k_{2} k_{3}$. Both transfer functions are expressed in $\mathrm{m} / \mathrm{V}$.

Using the parameter values mentioned previously, the step responses of both transfer functions are shown in Fig. 2. Observe the long settling time and very high overshoot due to the low damping factor of the mass-spring-damper system.

The Bode plot of the second transfer function is plotted in Fig. 3 as in the next section, a controller will be designed for the displacement of the second mass.

\section{CONTROLleR DESIGN}

In this section two types of controllers will be designed for the second mass of the system described in previous section with the purpose of comparing the performance of the designed controllers. Firstly, a model-based computer aided design technique (FRtool) will be used to design an integer-order proportional-integral (PI) controller. Secondly, a fractional order PI controller (FOPI) will be designed using model-based techniques.

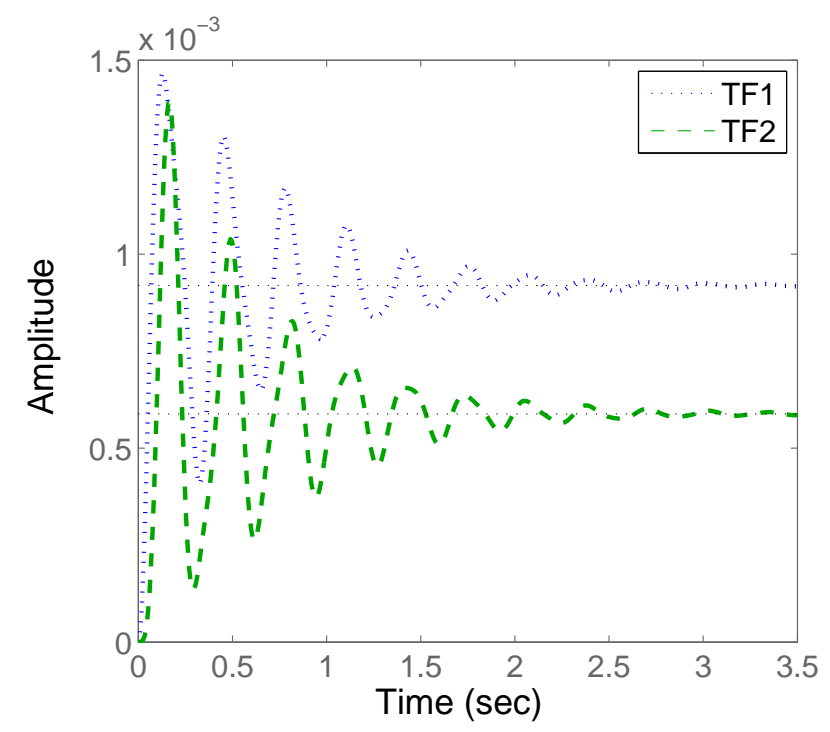

Fig. 2. Step responses for both transfer functions.

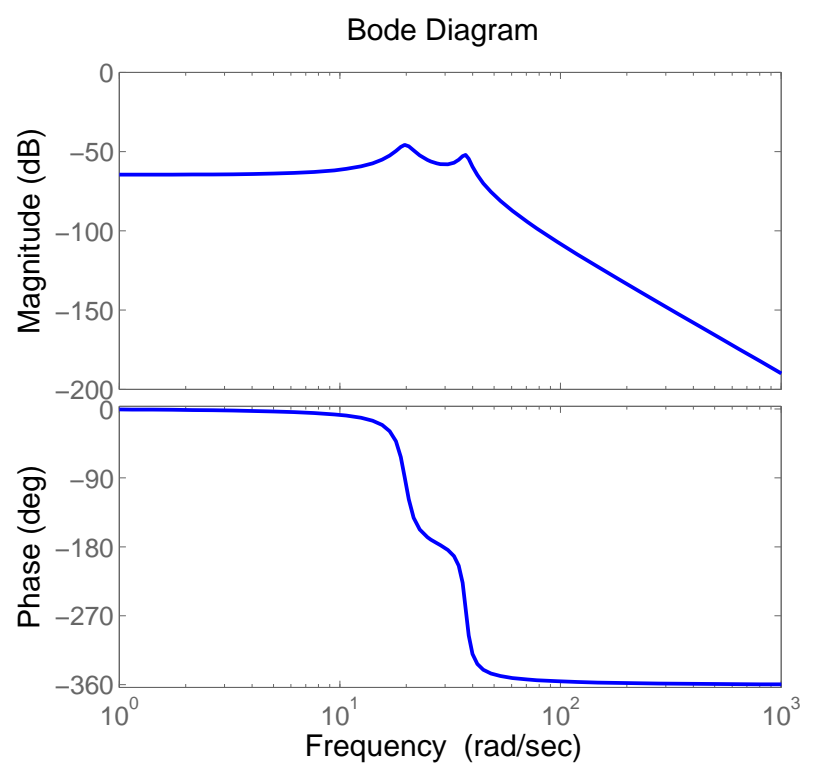

Fig. 3. Bode plot of the system describing the displacement of the second mass.

\section{A. Model-based integer-order controller design}

A model-based design technique called Frequency Response Toolbox (FRtool) [11], which uses information from the frequency response in the Nichols plot to design the parameters of the PI controller based on specifications such as settling time, \% overshoot, robustness, gain and phase margins is used. FRtool is a computer aided design (CAD) tool which uses the full knowledge of the process model. Other model-based design techniques can also be used to design PI controllers such as the Root Locus approach (RLtool) in MATLAB.

In FRtool, three different types of specifications are used to design a PI controller. The specifications used to design the current PI controller are: 
- $\quad$ Robustness $>0.4$,

- Settling time $<4 \mathrm{sec}$,

- $\%$ overshoot $<5 \%$.

In FRtool the PI is considered in the following form:

$$
P I(s)=K * \frac{(s-z)}{s}
$$

By playing with the position of the zero of the controller

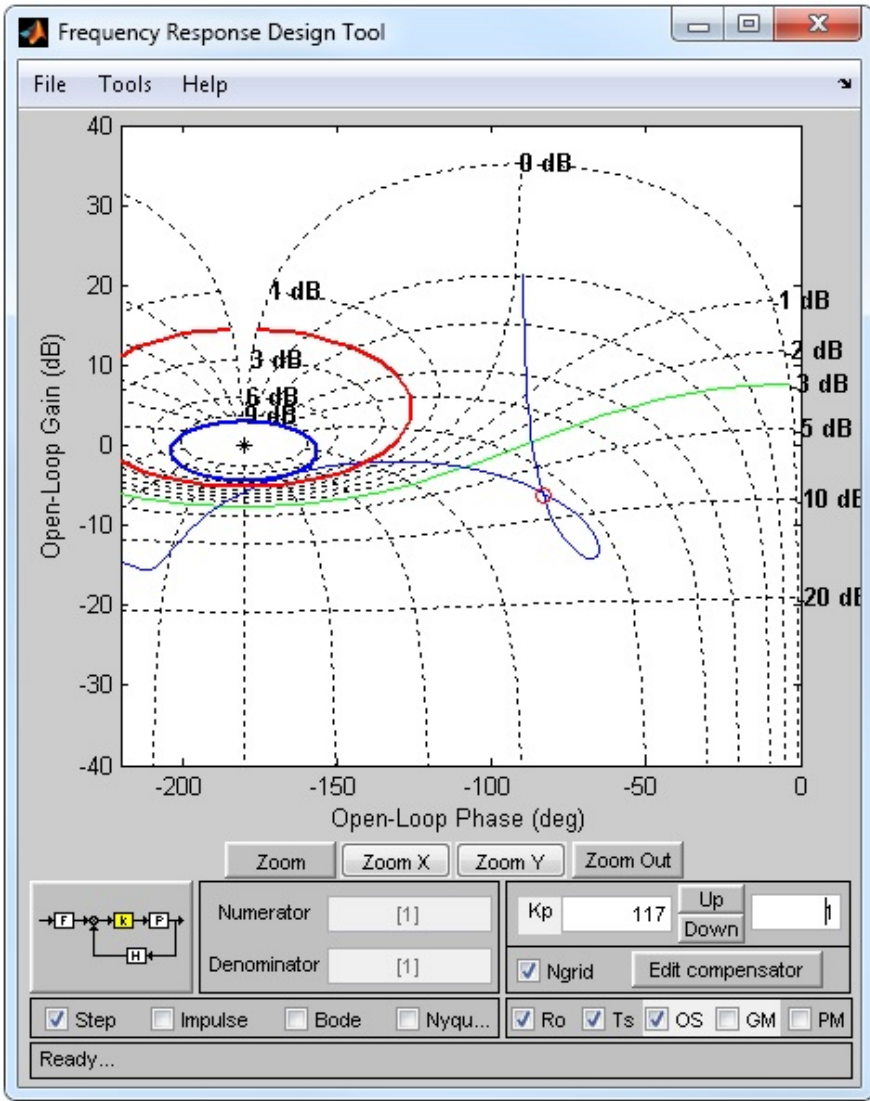

Fig. 4. Snapshot of the Nichols plot for controller design in FRtool.

and the controller gain, the shape of the open loop frequency response in the Nichols plot can be influenced. Fig. 4 shows the Nichols plot of the designed controller and the process. The thin blue line represents the open loop frequency response of the system. The thick blue line represents the robustness, the red line represent the M-circle which corresponds with the specified overshoot and the green line represents the $3 \mathrm{~dB}$ line in the Nichols plot. The zero of the designed PID controller has a value of -17 and the gain of the controller $K$ has a value of 117.

The resulting controller parameters are:

- $K_{p}=117$ and

- $T_{i}=0.059$

for the standard PID form:

$$
P I(s)=K_{p} *\left(1+\frac{1}{T_{i} s}\right)
$$

\section{B. Model-based fractional controller design}

FOPI controllers have the advantage of additional flexibility with respect to integer-order PI controllers. It is quite natural to conclude that the introduction of the fractional order $\lambda$ to the integral action in the $\mathrm{PI}^{\lambda}$ controller results in a more satisfactory compromise between positive and negative effects.

In model-based tuning techniques of FOPI controllers, methods to ensure certain specifications are used. In this paper, the used tuning method ensures specifications for gain crossover frequency, phase margin and zero steady state error [12].

The FOPI controller is expressed by:

$$
P I^{\lambda}(s)=C(s)=K_{p}\left(1+\frac{1}{T_{i} s^{\lambda}}\right)
$$

with $K_{p}, T_{i}$ and $\lambda$, the controller parameters.

The specification on the steady state error implies that $\lambda>$ 0 according to the Final-Value Theorem [13]. The condition on gain crossover frequency and phase margin can be both expressed by:

$$
T F\left(j \omega_{g c}\right) C\left(j \omega_{g c}\right)=-e^{j P M}
$$

with $P M$ the phase margin, $\omega_{g c}$ the gain crossover frequency, $T F\left(j \omega_{g c}\right)$ the transfer function of the system and $C\left(j \omega_{g c}\right)$ the transfer function of the controller.

When inserting (7) into (8), the following relation is obtained:

$$
C\left(j \omega_{g c}\right)=K_{p}\left(1+\frac{1}{T_{i}\left(j \omega_{g c}\right)^{\lambda}}\right)=\frac{-e^{j P M}}{T F\left(j \omega_{g c}\right)}
$$

Taking into account that $\left(j \omega_{g c}\right)^{\lambda}=\omega_{g c}^{\lambda} e^{j \frac{\pi \lambda}{2}}$, the values for $K_{p}$ and $T_{i}$ can be expressed as:

$$
T_{i}=\frac{-\Im\left(\frac{e^{j\left[P M+\frac{\pi \lambda}{2}\right]}}{T F\left(j \omega_{g c}\right)}\right)}{\sin \left(\frac{\pi \lambda}{2}\right)}
$$

For this system, the desired gain crossover frequency is $\omega_{g c}=$ $1.25 \mathrm{rad} / \mathrm{s}$ and the phase margin is taken to be $65^{\circ}$.

For any positive value of $\lambda$, a controller that achieves all three conditions can be found. The parameter $\lambda$ is tuned in order to increase the gain margin of the controlled system. Therefore, the gain margin of the controller system is expressed in function of $\lambda$ values between 0 and 2 (see Fig. 5). From this figure can be concluded that a maximal gain margin is obtained for $\lambda=1.3$. Notice that the fractional order is 


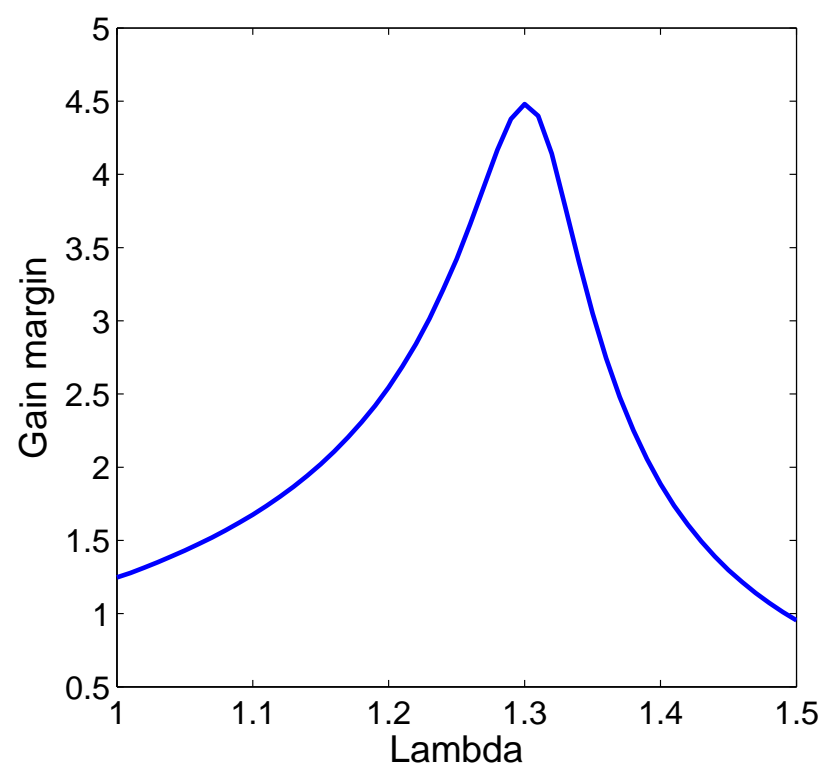

Fig. 5. Zoom in of the gain margin in function of $\lambda$.

higher than 1, which implies that there is an integrator in the transfer function of the controller. The corresponding values of $K_{p}$ and $T_{i}$ are respectively 91.44 and 0.04 .

\section{Simulations AND RESUlts}

A first simulation compares the performance between a PI controller and a FOPI controller for step inputs. Fractionalorder controllers have the inherent property of robustness to system gain variations. Therefore, a second simulation investigates the robustness of the designed controller to system gain variations.

\section{A. Implementation of FOPI controller}

Designing FOPI controllers is only one part of the dual problem in the theory of fractional-order controllers. Implementation of them is the most difficult problem to be solved. Although some references discuss hardware devices for fractional-order integrators [14], [15], these devices are restricted and difficult to tune. Alternatively, the authors choose to implement the FOPI controller by using a finite-dimensional integer-order transfer function. The fractional term $s^{\lambda}$ is then approximated by a finite-dimensional transfer function. The relative merits of the approximation method depend on the differentiation order and on whether an accurate frequency behavior is important.

The approximation method used in this research is the Modified Oustaloup Filter [16]. It fits the frequency response over a frequency range of interest $\left(\omega_{b}, \omega_{h}\right)$. The filter is expressed by:

$$
s^{\lambda} \approx\left(\frac{d \omega_{h}}{b}\right)^{\lambda}\left(\frac{d s^{2}+b \omega_{h} s}{d(1-\lambda) s^{2}+b \omega_{h} s+d \lambda}\right) \prod_{k=-N}^{N} \frac{s+\omega_{k}^{\prime}}{s+\omega_{k}}
$$

where the filter is stable for $\lambda \in(0,1), \omega_{k}^{\prime}=\omega_{b} \omega_{u}^{(2 k-1-\lambda) / N}$ and $\omega_{k}=\omega_{b} \omega_{u}^{(2 k-1+\lambda) / N}$. The parameters used for the approximation are: $N=6, \omega_{b}=10^{-3}, \omega_{h}=10^{3}, b=$ 10 and $d=9$. The Bode plots of the fractional-order PI controller and its integer-order approximation are shown in Fig. 6. In Fig. 7, the open loop of the compensated system can be seen for the fractional-order controller and its approximation. Notice that in the frequency range of interest $\left(10^{-3}, 10^{3}\right)$ the approximation is very good.

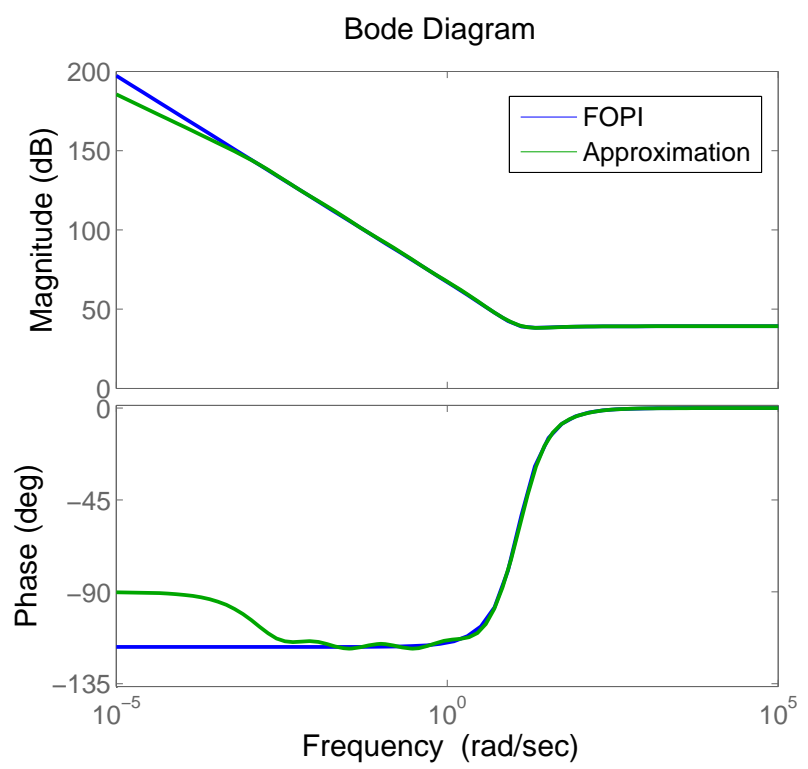

Fig. 6. Bode plots of the FOPI controller and its integer-order approximation.

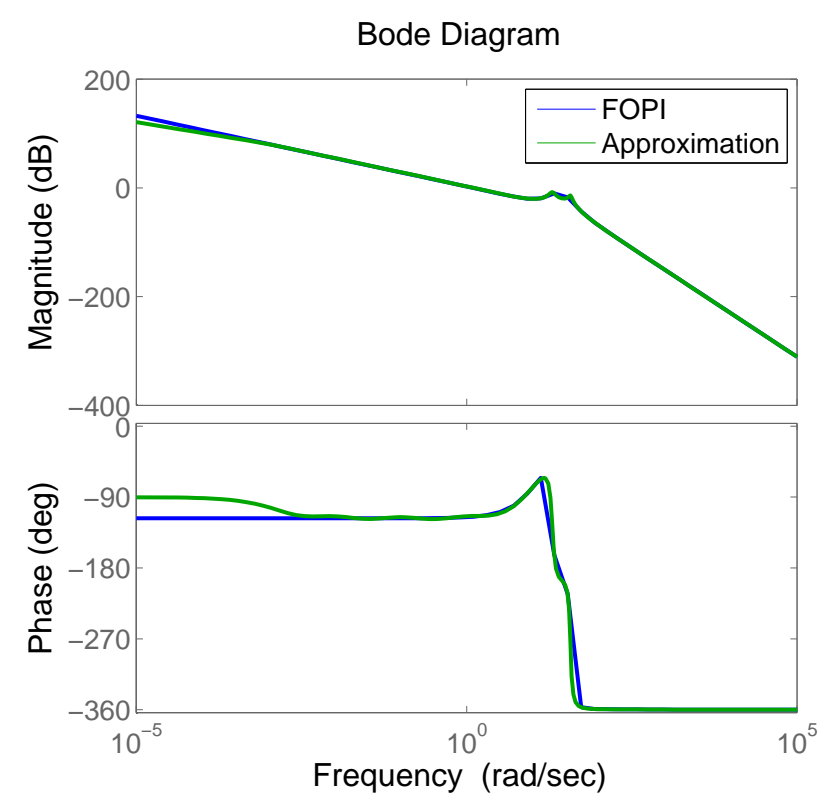

Fig. 7. Bode plots of open loop of the compensated system for the fractionalorder controller and its approximation.

The simplified approximated integer-order transfer function 
of the fractional-order controller is:

$$
\begin{aligned}
& C(s)=\frac{91.44(s+5.92)(s+0.45)(s+0.04)(s+0.004)}{s(s+0.002)(s+0.02)(s+0.22)(s+2.24)} . \\
& \frac{(s+220.7)(s+854.2)\left(s^{2}+24.65 s+222.5\right)}{(s+22.39)(s+223.9)(s+854.7)}
\end{aligned}
$$

Notice that this is a higher order transfer function of order 8 .

\section{B. Comparison between PI controller and FOPI controller}

In order to compare the performance of the PI controller and the FOPI controller, a simulation in MATLAB/SIMULINK is performed where the Simulink scheme is shown in Fig. 8. The results of the simulation are shown in Fig. 9

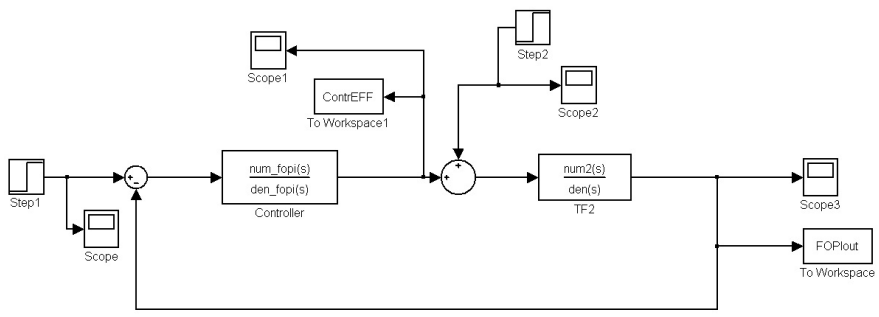

Fig. 8. Simulink scheme for step inputs.
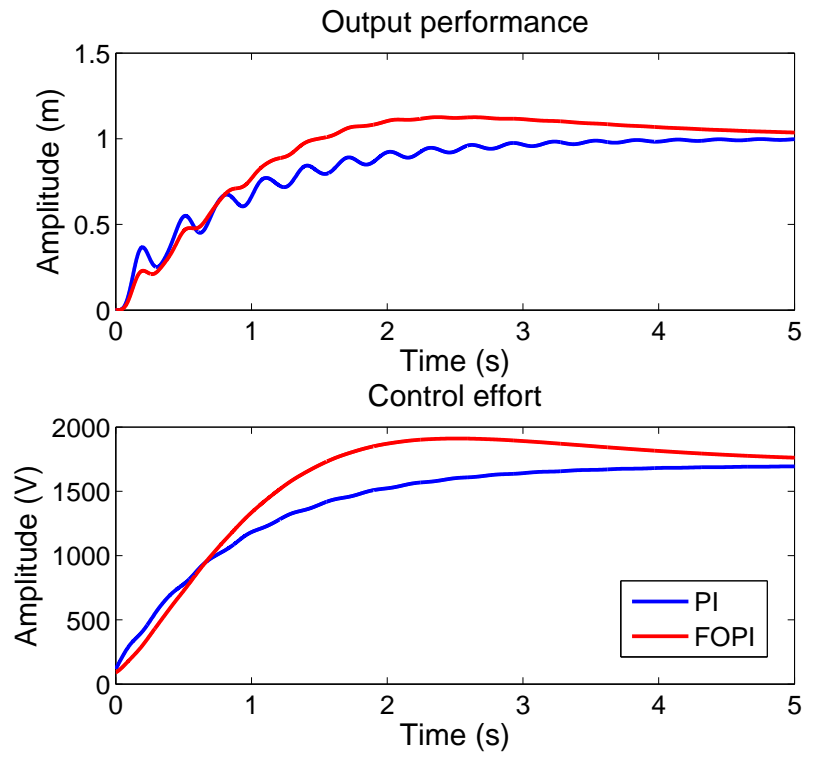

Fig. 9. Step responses of the closed loop system.

It can be observed that the FOPI controller provides a smooth performance at a similar control effort to that of the PI controller (i.e. no oscillations in the response). The explanation for the absence of the oscillations in the case of the FOPI controller is twofold. In order to be implemented, the FOPI controller has to be approximated by a high-order integer order transfer function, in this case of order 8 [12]. Hence, the first explanation is that the degree of the FOPI controller is finally much higher than that of the classical PI controller. Therefore, more zeros and poles can compensate for the oscillatory dynamics of the system, as observed from Fig. 10 which depicts the root loci for the two designs. The second explanation is that indeed, the extra zeros in the approximated transfer function of the FOPI controller will compensate the complex conjugated poorly damped poles of the plant.
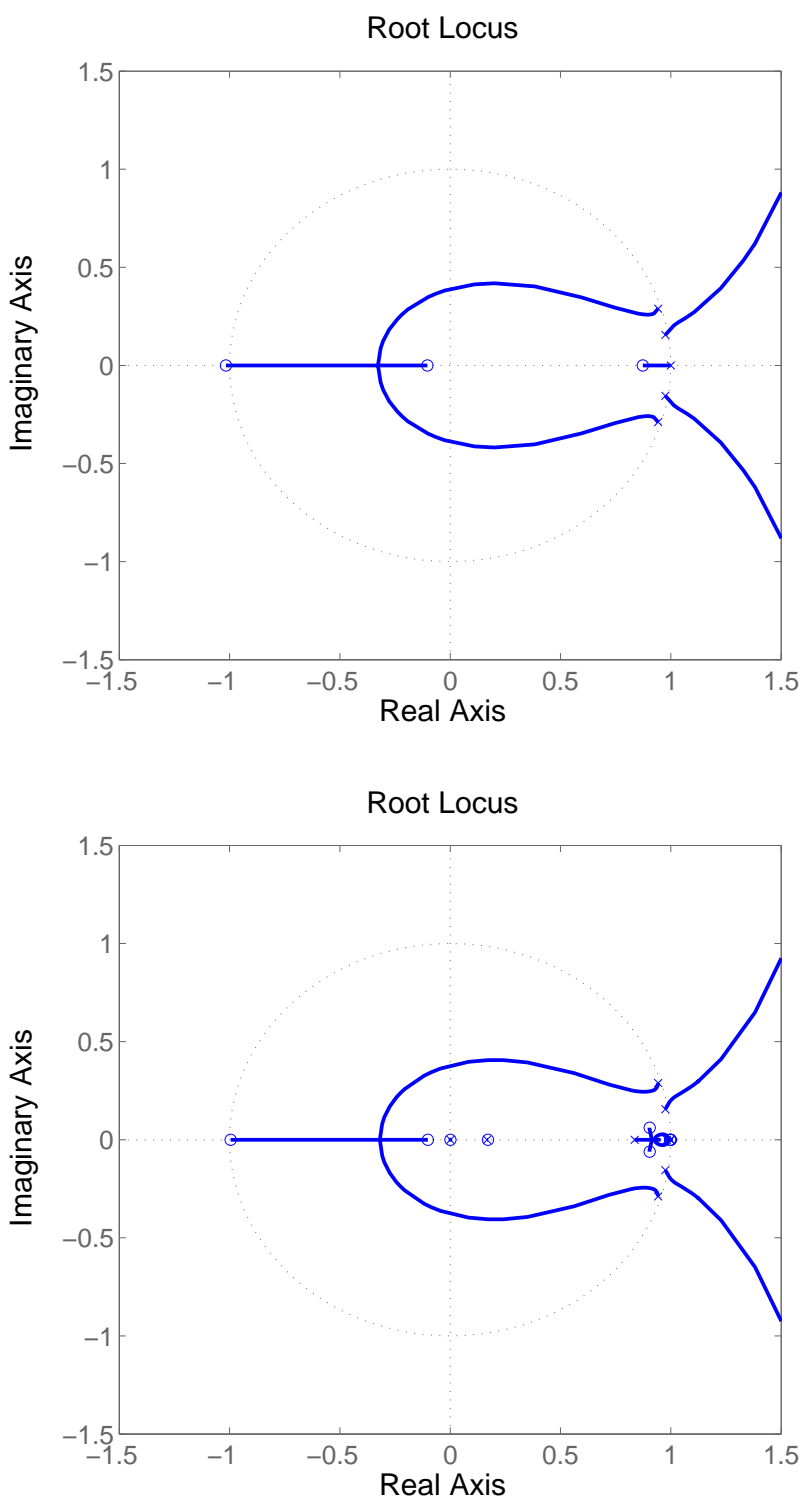

Fig. 10. Root Locus of the discretized closed loop for the integer-order PI controller (top) and the fractional-order FOPI controller (bottom).

\section{Robustness to gain variation}

Modeling errors can introduce a change in the system's gain. In order to investigate the robustness of the designed controller to such changes, the gain of the system is varied. The static gain of the motor in the system $K$ is nominal taken to be 1 . The nominal gain is varied by $20 \%$ in both directions, i.e. $80 \%$ of the nominal gain and $120 \%$ of the nominal gain. The resulting output signals and control efforts are shown in Fig. 11. 

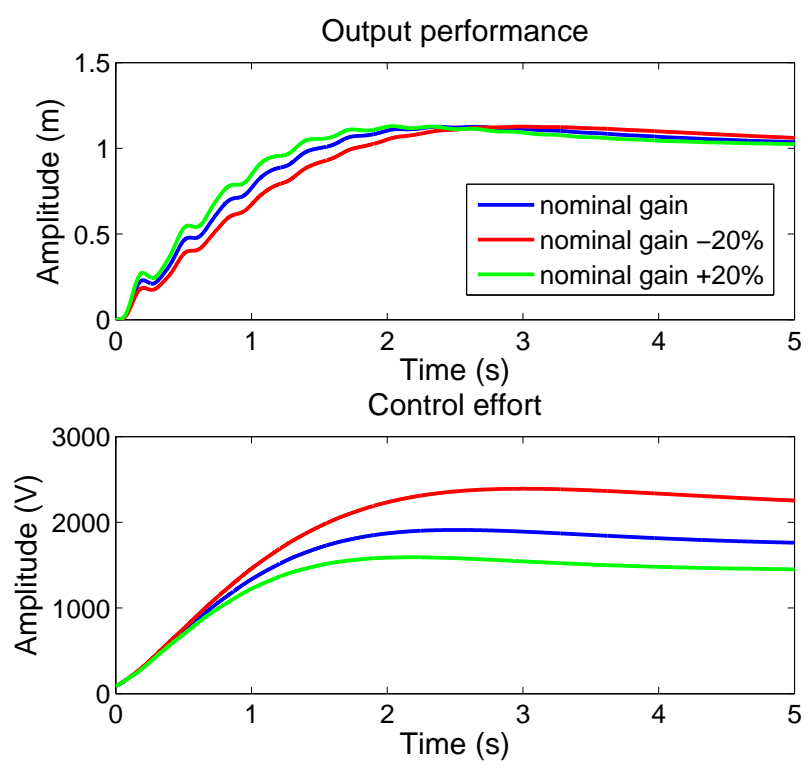

Fig. 11. Step responses of the closed loop system for robustness simulation.

Notice from Fig. 11 that the system is indeed robust to gain changes. The output responses do not vary much even with gain changes of $20 \%$. Note, however, that the control effort changes when the gain is varied.

\section{CONCLUSION}

Based on the results, it can be concluded that a fractional order controller might pose interesting advantages over the classical integer order control. Currently, efforts are being made to test these controllers on the real setup. Simulations confirm the theoretical insight that a fractional PI controller has an inherent robustness to gain variations in poorly damped electromechanical systems. Future work includes obtaining experimental data to confirm the simulation results.

\section{ACKNOWLEDGMENT}

Clara M. Ionescu is a post-doc fellow of the Research Foundation - Flanders (FWO).

\section{REFERENCES}

[1] M. A. Rahimian, M. S. Tavazoei and F. Tahami. Fractional-order PI Speed Control of a two-mass drive system with elastic coupling, Proceedings of FDA'10. 4th IFAC Workshop Fractional Differentieaion and its Applications, 2010.

[2] C. Poussot-Vassal, C. Spelta, O. Sename, S.M. Savaresi and L. Dugard. Survey and performance evaluation on some automotive semi-active suspension control methods: A comparative study on a single-corner model, Annual Reviews in Control, vol. 36, pp. 148-160, 2012.

[3] B. M. Nigg and W. Liu. The effect of muscle stiffness and damping on simulated impact force peaks during running, Journal of Biomechanics, vol. 32(8), pp. 849-856, 1999.

[4] P. Gardonio, S. Miani, F. Blanchini,D. Casagrande and S.J. Elliott. Plate with decentralised velocity feedback loops: Power absorption and kinetic energy considerations, Journal of Sound and Vibration, vol. 331(8), pp. 1722-1741, 2012

[5] I. Podlubny. Fractional-order systems and $\mathrm{PI}^{\lambda} \mathrm{D}^{\mu}$ controllers. IEEE Transactions on Automatic Control, vol. 44, pp. 208-214, 1999.

[6] S. H. HosseinNia, I. Tejado and B. M. Vinagre. Fractional-order reset control: Application to a servomotor, Mechatronics, vol. 23(7), pp. 781788, 2013

[7] C.A. Monje, B.M. Vinagre, V. Feliu and Y.Q. Chen. Tuning and autotuning of fractional-order controllers for industry applications. Control Engineering Practice, vol. 16(7), pp. 798-812, 2008.

[8] D. Valerio and J. Sa da Costa. Tuning of fractional PID controllers with Ziegler-Nichols-type rules. Signal Processing, vol. 86, pp. 2771-2784, 2006.

[9] Y. Q. Chen, T. Bhaskaran, and D. Xue. Practical tuning rule development for fractional order proportional and integral controllers. ASME Journal of Computational and Nonlinear Dynamics, vol. 3, pp. 02140310214037, 2008

[10] A. Oustaloup. La dérivation non entière, Hermès , Paris, 1995.

[11] R. De Keyser and C. M. Ionescu, FRtool: A frequency response tool for CACSD in Matlab, IEEE International Symposium on Computer Aided Control Systems Design, pp. 2275-2280, Munich 2006.

[12] C.A. Monje, Y.Q. Chen, B.M. Vinagre, D. Xue and V. Feliu. Fractionalorder Systems and Controls: Fundamentals and Applications, SpringerVerlag London, 2010.

[13] K. Ogata. Modern Control Engineering, 4th edition, Prentice Hall, 2001.

[14] I. Petrás̃, I. Podlubny, P. O'Leary. Analogue Realization of Fractional Order Controllers. Fakulta BERG, TU Kos̃ice, 2002.

[15] G.W. Bohannan. Analog realization of a fractional controller, revisited. In: BM Vinagre, YQ Chen, eds, Tutorial Workshop 2: Fractional Calculus Applications in Automatic Control and Robotics, Las Vegas, USA, 2002.

[16] D.Y. Xue, C.N. Zhao, Y.Q. Chen. A modified approximation method of fractional order system. Proceedings of IEEE Conference on Mechatronics and Automation. Luoyang, China, pp. 1043-1048, 2006. 